\title{
A Fast Running Test Bed Model to Evaluate Atmospheric Plume Source Properties. I: Initial Test Scenario
}

A.S. Grossman, C.F. Molenkamp, K.E. Grant

\section{June 1, 2001}

U.S. Department of Energy

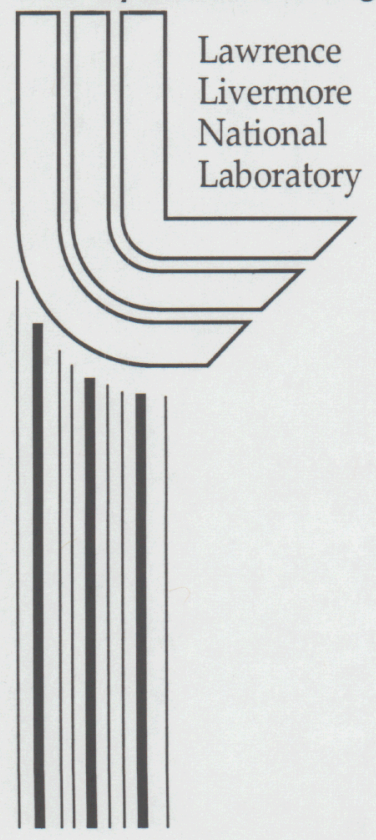




\section{DISCLAIMER}

This document was prepared as an account of work sponsored by an agency of the United States Government. Neither the United States Government nor the University of California nor any of their employees, makes any warranty, express or implied, or assumes any legal liability or responsibility for the accuracy, completeness, or usefulness of any information, apparatus, product, or process disclosed, or represents that its use would not infringe privately owned rights. Reference herein to any specific commercial product, process, or service by trade name, trademark, manufacturer, or otherwise, does not necessarily constitute or imply its endorsement, recommendation, or favoring by the United States Government or the University of California. The views and opinions of authors expressed herein do not necessarily state or reflect those of the United States Government or the University of California, and shall not be used for advertising or product endorsement purposes.

This work was performed under the auspices of the U. S. Department of Energy by the University of California, Lawrence Livermore National Laboratory under Contract No. W-7405-Eng-48.

This report has been reproduced directly from the best available copy.

Available electronically at http://www.doe.gov/bridge

Available for a processing fee to U.S. Department of Energy and its contractors in paper from

U.S. Department of Energy Office of Scientific and Technical Information

P.O. Box 62

Oak Ridge, TN 37831-0062

Telephone: (865) 576-8401

Facsimile: (865) 576-5728

E-mail: reports@adonis.osti.gov

Available for the sale to the public from

U.S. Department of Commerce

National Technical Information Service 5285 Port Royal Road Springfield, VA 22161

Telephone: (800) 553-6847

Facsimile: (703) 605-6900

E-mail: orders@ntis.fedworld.gov

Online ordering: http://www.ntis.gov/ordering.htm

OR

Lawrence Livermore National Laboratory Technical Information Department's Digital Library http://www.llnl.gov/tid/Library.html 


\title{
A FAST RUNNING TEST BED MODEL TO EVALUATE ATMOSPHERIC PLUME SOURCE PROPERTIES. I: INITIAL TEST SCENARIO
}

\author{
Allen S. Grossman \\ Charles F. Molenkamp \\ Keith E. Grant
}

This work was performed under the auspices of the U.S. Department of Energy by the University of California Lawrence Livermore National Laboratory under Contract No. W-7405-Eng-48. 


\begin{abstract}
Given an unknown but detected release of a toxic agent, the current NARAC capability for reconstructing source characteristics is a highly manual procedure that often relies on analyst judgement and requires many hours of computations for a refined analysis. There is no automated, optimization approach to estimating the source characteristics. A fast running, prototype atmospheric inversion model has been developed for use as a test bed for the evaluation of source inversion schemes. The model was applied to a simple puff release scenario to test the relationship between the amount of sampled data obtained and the accuracy of the determination of the inverted source parameters. The initial inversion scheme chosen for the test bed model utilizes the Marquardt method coupled to a Gaussian puff atmospheric dispersion model driven by a COAMPS model wind field. The inversion scheme results are used in conjunction with a sensor realization probability model for a sensor realization scenario consisting of 1000 possible sensor realizations. The results of the initial test calculations indicate that the inversion procedure produces good results for the four source parameters, location (x,y), release time, and strength along with reasonably well defined maximum probabilities for the sensor realization scenarios. The model runs relatively fast, taking $~ 100$ seconds per inversion on a Sparc 10 workstation.
\end{abstract}




\section{INTRODUCTION}

Given an unknown but detected release of a hazardous material, the current NARAC capability for reconstructing the material source using downwind sensors is a highly manual procedure that often relies on analyst judgement and requires several hours of computations for a refined analysis. There is no automated, optimization approach to estimating the source characteristics from observational data. Delays in source characterization can degrade the ability of the modeling system to provide adequate forward-time concentration predictions.

Optimal inversion of observational data to determine source characteristics has been extensively treated in the scientific literature (Bevington and Robinson, 1992 [BR]; Lloyd, 1984; Barker et al., 1995; Flesch et al., 1995, Kasibhatla, 2000). There are many optimal inversion schemes which could be used to augment the current NARAC capabilities for agent source characterization. Two representative schemes which appear to be particularly suitable for NARAC applications are the Marquardt Non Linear Least Squares method (Edwards et al., 1992) and the Bayesian Probability Update Method (Barker et al. 1995). These are both forwardtime schemes.

With the Marquardt method, the error cost functional is minimized by setting its first derivative with respect to the assumed source properties to zero and then iteratively solving for corrections to the assumed source properties. This method requires derivatives of the dispersion model with respect to the assumed source parameters, and this is usually done numerically by performing many individual parameter model sensitivity runs over the space of input parameters.

The Bayesian method is based on a set of probabilities of possible model states determined prior to observational data collection (priors). Once observational data is obtained, the method provides a prescription to calculate a new set of probabilities taking into account the observed data. Each new set of data leads to an updated probability set. The method requires many forward runs of the dispersion model to determine state probabilities.

A promising approach to determine feasible source properties which can then be used as the starting points for forward dispersion schemes has been considered by Flesch et al. (1995). The method utilizes a "backward" dispersion model, starting from the observation point, to determine areas where the presently observed pollutant particles may have originated. This method can be computationally intensive when large numbers of sensors are involved, and is therefore most promising for a limited number of sensors.

Other major factors that are important to consider in the data inversion methodology are the characterization and incorporation of the effects of meteorological uncertainties on mean wind fields and turbulent fluctuations in sensor concentration observations. Mean wind field forecasts on a regional scale can vary dramatically due to uncertainties in the initial and boundary conditions (Leach and Chin, 2000) and mean wind forecast uncertainties must be characterized in order to compare forecasts with observations. In addition, estimates of turbulent concentration fluctuations (given the mean wind) must be made in order to compare ensemble-mean, modelpredicted air concentration fields to sensor measurements which will likely be individual realizations of the possible concentration fields. Methods for estimating concentration fluctuations have been developed (e.g.,Yee et al., 1998) and need to be incorporated in the data inversion schemes. 
The major purpose of this report is to describe a fast running, prototype atmospheric inversion model that will be used to evaluate source inversion schemes. The model will be applied to a simple puff release scenario to test the relationship between the amount of data obtained and the accuracy of the determination of the inverted source parameters. The initial inversion scheme chosen for the test bed model uses the Marquardt method coupled to a Gaussian Puff atmospheric dispersion model, INPUFF (Peterson and Lavdas, 1986).

\section{INVERSION METHODOLOGY}

Following $\mathrm{BR}$ we can write a probability function which relates the probability $\mathrm{P}_{\mathrm{i}}$ of an observation $\mathrm{y}_{\mathrm{i}}$ about the actual value $\mathrm{y}_{0}$ at location $\mathrm{x}_{\mathrm{i}}$. Assuming that the sensor observations are drawn from a Gaussian distribution with a mean value of $y_{0}$ and a standard deviation $\sigma_{i}$, the probability density function is given by

$$
P_{i}=\left\{1 /\left[\sigma_{i} \sqrt{ }(2 \pi)\right]\right\} \exp \left[-0.5\left(\left[y_{i}-y_{0}\right] / \sigma_{i}\right)^{2}\right] .
$$

If it can also be assumed that the value of $y_{0}$ can be estimated via an atmospheric dispersion model as,

$$
\mathrm{y}_{0}=\mathrm{f}\left(\mathrm{x}_{\mathrm{i}}, \mathrm{t}_{\mathrm{i}}, \mathrm{a}_{1} \ldots \ldots \ldots \ldots \ldots \mathrm{a}_{\mathrm{n}}\right) \text {, }
$$

where $x_{i} / t_{i}$ are the location/time of the observation, and the a's are various source parameters which include location and strength, then the probability of the particular combination of sensor reading and model realization can be expressed by Eq. 1. It may not always be possible to estimate the actual mean value of a set of observations, $y_{0}$, with the estimated mean value from a simple dispersion model and in that case a different form of Eq. 1 must be utilized. However, for the purposes of the test calculations to be performed in this paper, the above assumption will be made. When there are multiple sensor readings for different locations and times, the probability density of the realization encompassing all the sensor readings is

$$
\begin{aligned}
& P=\Pi\left(P_{i}\right), \\
& =\Pi\left(1 /\left[\sigma_{i} \sqrt{ }(2 \pi)\right]\right) \exp \left[-0.5 \Sigma\left(\left[y_{i}-y_{0}\right] / \sigma_{i}\right)^{2}\right] .
\end{aligned}
$$

This function is known as the probability function or likelihood function (BR). In order to maximize the likelihood function with respect to the parameters $a_{1} . . a_{n}$ it is necessary to minimize the exponential term in Eq. 3;

$$
\mathrm{X}^{2} \equiv \Sigma\left\{\left(\left[\mathrm{y}_{\mathrm{i}}-\mathrm{y}_{0}\right] / \sigma_{\mathrm{i}}\right)^{2}\right\}
$$

This can be accomplished via a manual grid search procedure or an automated grid search method. One automated method utilizes a Taylor expansion of the $\mathrm{X}^{2}$ function about an initial set of parameters and then solves for the increments to the initial set to minimize the function (linearization of the fitting function). Another automated method utilizes a gradient search method (steepest descent). All initial parameters are given an increment based on the gradient of the $\mathrm{X}^{2}$ function at the initial guess point. The Marquardt scheme combines both of the above 
approaches in a way that uses the gradient method when far from the minimum of the $\mathrm{X}^{2}$ function and the linearized fitting method when close to the minimum.

The Marquardt scheme requires an initial guess for the model parameters which is reasonably close to the true parameters so that the iterative minimization procedure is convergent. This initial guess can be determined using a manual grid search method. In particular, when the source location coordinates are included in the model parameter set to be determined, a 'backtrack' dispersion model has been developed which provides a considerable reduction in the initial guess grid search. Assuming a particular release time value, the backtrack/grid search technique estimates source location by calculating a backwards trajectory, from the sensor, for each non-zero concentration sample and then calculates the average or concentration weighted average (centroid) location. Sample calculations, to be discussed later, show that the 'backtrack' method is very efficient at providing starting parameter locations for the Marquardt inversion scheme.

For the calculations to be described in this report it was decided to use the log of the concentration $\left(\mathrm{y}_{\mathrm{i}}, \mathrm{y}_{0}\right)$ as the primary concentration variable. This was done to prevent the highest observed concentrations from totally dominating the $\mathrm{X}^{2}$ function and to avoid negative concentrations in the distribution of sensor readings.

\section{INPUFF MODEL}

The INPUFF (INtegrated PUFF, Petersen and Lavdas, 1986) dispersion model is a fast running semi-analytic model designed to simulate the dispersion from semi-instantaneous sources over a spatially and temporally variable wind field. The dispersion scheme is based on the Gaussian puff assumptions (Pasquill and Smith, 1983, Turner, 1994). The instantaneous concentration downwind from a release height, $\mathrm{H}$, is given by the expression:

$$
\begin{gathered}
\mathrm{C}(\mathrm{x}, \mathrm{y}, \mathrm{z}, \mathrm{H})=\mathrm{Q}_{\mathrm{T}} /\left[(2 \pi)^{3 / 2} \sigma_{\mathrm{x}} \sigma_{\mathrm{y}} \sigma_{\mathrm{z}}\right] \exp \left[-(\mathrm{x}-\mathrm{ut})^{2} / 2 \sigma_{\mathrm{x}}^{2}\right] \\
\exp \left[-\mathrm{y}^{2} / 2 \sigma_{\mathrm{y}}^{2}\right]\left\{\exp \left[-(\mathrm{z}+\mathrm{H})^{2} / 2 \sigma_{\mathrm{z}}^{2}\right]+\exp \left[-(\mathrm{z}-\mathrm{H})^{2} / 2 \sigma_{\mathrm{z}}^{2}\right]\right\},
\end{gathered}
$$

where $C$ is the downwind concentration, $Q_{T}$ is the total mass of the release, the $\sigma$ 's define the plume spread, and $\mathrm{u}$ is the horizontal velocity. The coordinates $\mathrm{x}, \mathrm{y}$, and $\mathrm{z}$ define the downwind distance, the crosswind distance, and the altitude above ground. For the problems considered here $\sigma_{x}=\sigma_{y}$, and $\sigma_{y}$ is a function of the downwind distance, the meteorological stability conditions, and the release height. A continuous release is represented by a series of instantaneous puffs. The INPUFF model has the following capabilities;

a. Single or multiple point sources at up to 1000 locations,

b. Up to 144 meteorological periods of the same time length,

c. Wind fields for each meteorological period for up to 100 user-defined grid locations,

The most recent release of the INPUFF model allows the above numerical limits to be changed via input file specification. The model also has the following limitations:

a. Wind direction constant with height,

b. No consideration of chemical reactions,

c. No explicit treatment of complex terrain other than implied in the wind field calculations.

The INPUFF model has many input parameters relating to different atmospheric conditions and source configurations. For the calculations presented here a relatively simple set of input parameters (described below) was chosen for the evaluation of the prototype inversion model. 


\section{TEST SCENARIO}

A test scenario, based on a puff release of material from the Salt Lake City Utah area, was used to evaluate the inversion model. In the test scenario a set of sensors are uniformly distributed in the test area. Sensor readings are generated from assumed source parameters and INPUFF simulations. The inversion model is then used to calculate the source parameters from the sensor readings. The inverted source properties are then compared to the actual values. The inversion scheme results will be used in conjunction with a sensor realization probability model for a sensor realization scenario consisting of 1000 possible sensor reading realizations. An hourly wind field, for use by the dispersion model, was generated by the LLNL version of the Naval Research COAMPS regional prognostic model (Hodur, 1997) for a $40 \mathrm{~km} \mathrm{x} 40 \mathrm{~km}$ domain surrounding Salt Lake City Utah. The resolution of the wind grid was $4 \mathrm{~km}(\mathrm{EW}) \mathrm{x} 4 \mathrm{~km}$ (NS). Inspection of the COAMPS meteorology indicated a wind flow approximately west to east. The source was located on the west side of the domain approximately mid way between the north and south sides of the grid. The sensor readings (truth) were generated by calculation of the time variation of the concentration at each sensor using the dispersion model with the actual source parameters. A fixed sensor integration time of five minutes was chosen for the test scenario, providing twelve sensor readings per hour.

The source parameters selected for the test scenario are:

a. Source horizontal location $(x-y)(\mathrm{km}), x=2 \mathrm{~km}, y=20 \mathrm{~km}$.

b. Source release start time $(t)(\mathrm{sec}), t=3600 \mathrm{~s}$.

c. Source strength $(\mathrm{Q})(\mathrm{g} / \mathrm{s}), \mathrm{Q}=100 \mu \mathrm{g} / \mathrm{s}$.

d. Puff duration $=6 \mathrm{~s}$ ( (single puff).

Auxiliary parameters selected for the INPUFF model are:

a. Source release height $=1 \mathrm{~m}$.

b. Air Temperature $=296 \mathrm{~K}$.

c. Stability Class $=3$ ( P-G Class C, particular relation for $\sigma$ vs. downwind distance).

d. Mixing height $=1000 \mathrm{~m}$.

Sensor placement (12 sensors at $8 \mathrm{~km}$ intervals) for the test runs was;

$$
\begin{aligned}
& \text { a. } x=3,11,19,27 \mathrm{~km} \\
& \text { b. } y=12,20,28 \mathrm{~km} .
\end{aligned}
$$

Figure 1. shows the wind vector field over the $40 \mathrm{~km} \mathrm{x} 40 \mathrm{~km}$ domain superimposed on a map of the one hour integrated pollutant concentrations calculated by the INPUFF model for a hiresolution sensor field spacing of $\mathrm{x}=1 \mathrm{~km}$ and $\mathrm{y}=1 \mathrm{~km}$ (used for plotting the figure, not for generating hypothetical sensor readings for the inversion model). 


\section{PROBABILITY OF INDIVIDUAL AND CUMULATIVE SENSOR READING REALIZATIONS}

Although an inversion model can determine the source parameters for a set of sensor readings, the uncertainty in these sensor readings will cause uncertainty in the evaluation of the source parameter set. Uncertainties in the evaluation of the source parameter set can also be caused by stochastic uncertainties in the physical properties in the dispersion model, an example of which would be the uncertainty in the atmospheric turbulence parameterization. As a first exercise, this study will be concerned with uncertainty in sensor readings since this can be readily expressed in an analytic form. The procedure is as follows: the non-zero "truth" concentrations are perturbed with random errors to provide a set of simulated observations, $\mathrm{C}_{0}$. The inversion technique uses these observations to estimate the source properties; it does not know about the "truth" sensor readings. A realization is defined as the set of non zero sensor readings (both spatially and temporally) where each individual sensor reading includes a random perturbation from the "truth" value. We assume the perturbed sensor readings follow a log normal probability distribution about the "truth" concentration with an assumed standard deviation of $\alpha$. Each perturbed sensor reading will then have a probability based on a log normal distribution. The probability of the realization is the product of the individual sensor probabilities divided by the product of the probability of the realization where each sensor reads the "truth" run values. An inversion (Section II. above) is performed for each realization (1000 realizations are used to determine the source parameter probability distribution). Calculations will be presented for two values of $\alpha(2,4)$ to illustrate how the uncertainty in the sensor readings translates to uncertainty in the inverted source properties.

Actual sensor perturbations can be generated in two ways. The method actually used for the calculations presented here (Sample 1) consists of a set of sensor realizations generated using a normal random number generator. With this method, each set of sensor readings, as well as the distribution of realizations, obeys a normal frequency distribution. An alternative method (Sample 2) would utilize a random number generator to determine a perturbation to the actual sensor reading and then assign a probability, based on the log normal distribution, to the individual perturbed sensor reading (Iman and Shortencarier, 1984). The first method automatically takes the sensor reading probabilities into account while the second method would provide more of the realizations in the wings of the probability distribution.

In order to calculate the probability of the source location at any particular $x-y$ point, the $x-y$ plane is divided into squares $0.1 \mathrm{~km}$ on a side, centered on the true source location. The number of sensor realizations for which the source location occurs in a particular $x-y$ square is tabulated. For the Sample 1 method, the number of source locations in each square divided by the total number of sensor realizations (normalization factor) is interpreted as the probability of the source location in that square. Using the Sample 2 method, the realization probabilities for each source location within a particular $x-y$ square would be summed to obtain the cumulative probability for the source location in the square. This probability is normalized by dividing by the probability of the "truth value" sensor realization. Since the major concern is the relative probabilities square to square, the normalization factors are of minor consequence. The estimated release time and source strength were obtained in the Sample 1 method by calculating the mean values in the $x-y$ square where the source location occurred most often. The same quantities could be obtained in the Sample 2 method by calculating the probability weighted mean value for release time and source strength in the $x-y$ square having the highest source location probability. 


\section{RESULTS AND DISCUSSION}

An inversion calculation begins with a modified grid search to determine an initial guess for the four source variables $(x, y, t, Q)$ which is then provided to the inversion algorithm. The modified grid search is done in the following manner. First, a set of $t$ and $Q$ values are chosen within the expected range of the actual release values. Second, the backtrack method is used to determine the source location $(x, y)$ for each $t, Q$ value pair. The INPUFF model is then run in the forward direction for the assumed source and the $\mathrm{X}^{2}$ values are then determined for each $t, Q$ value pair. The $t, Q$ value pair and associated $x, y$ source location with the minimum $\mathrm{X}^{2}$ is used as the initial guess for the inversion algorithm. The inversion scenario described above was calculated for two values of the log normal sensor reading variation parameter, $\alpha=2$ and 4 . There were 12 non-zero sensor readings obtained for the sensor array over the one hour test period for any particular sensor realization. The summary of the inversion results is given in Table 1. The "truth values" of the source parameters are shown along with a) the inverted $x$ and $y$ values of the 100 meter square box where the inversion source occurs most often, b) the inverted release time value in the 10 second interval which occurs most often in the $x-y$ box with the maximum source locations, and c) the inverted source strength value in the $10 \mu \mathrm{g} / \mathrm{s}$ interval which occurs most often in the $x-y$ box with the maximum source locations, for each $\alpha$ value and each of the backtrack methodologies (CEN and AV). The figures shown in parentheses indicate the maximum probability associated with the inverted source properties. Figure 2a shows the source location points for the 1000 realizations in the $x$-y plane for the $\alpha=2$, Sample 1 methodology. The "+" signs are the locations obtained using the concentration weighted average backtrack method (CEN) and the " $x$ " signs are the locations using the straight average backtrack method (AV). Figure $2 b$ shows the results for the $\alpha=4$ case. These results show a relatively tight clustering of source locations about the actual point $(x=2 \mathrm{~km}, y=20$ $\mathrm{km}$ ). Also secondary, clustering can be seen at $x \sim 2.1 \mathrm{~km}$, and $y \sim 20.3 \mathrm{~km}$. The clustering is, as expected, tighter for the $\alpha=2$ case than for the $\alpha=4$ case because of the narrower sensor perturbation range. This can be clarified by examination of the probability histograms in the $x-y$ plane for squares of $100 \mathrm{~m}$ per side centered about the actual source point. These histograms (number of times predicted source was in each square) are shown in Figures 3a (AV) and $3 \mathrm{~b}(\mathrm{CEN})$ for the $\alpha=2$ case and Figures 3c (AV) and 3d (CEN) for the $\alpha=4$ case. Contour plots of these histograms are shown in Figures $4 \mathrm{a}(\mathrm{AV})$ and $4 \mathrm{~b}(\mathrm{CEN})$ for the $\alpha=2$ case and Figures $4 \mathrm{c}(\mathrm{AV})$ and $4 \mathrm{~d}(\mathrm{CEN})$ for the $\alpha=4$ case. For both $\alpha$ cases, CEN source locations produce a double peaked probability distribution while the AV source locations produce a single peaked probability distribution. For the $\alpha=2$, AV case the peak probability of 0.341 occurs in the box centered at $x=2.0 \mathrm{~km}, y=20 \mathrm{~km}$, with a half width of $\sim 1 \mathrm{~km}$ and the 90 percent probability contour enclosed within the area $x=1.8-2.2 \mathrm{~km}, y=19.8-20.3 \mathrm{~km}$. For the $\alpha=2$, CEN case a double probability peak occurs. The maximum peak probability of 0.233 occurs in the box centered at $x=2.0 \mathrm{~km}, y=20 \mathrm{~km}$, while a secondary probability peak of value 0.119 occurs at $x=2.1 \mathrm{~km}, y=20.3 \mathrm{~km}$. The 90 percent probability contour is enclosed within the area $x=1.8-2.2 \mathrm{~km}, y=19.8-20.4 \mathrm{~km}$. For the $\alpha=4$, AV case the peak probability of 0.104 occurs in the box centered at $x=2.0 \mathrm{~km}, y=20 \mathrm{~km}$, with a half width of $\sim 3 \mathrm{~km}$ and the 90 percent probability contour enclosed within the area $x=1.7-2.3 \mathrm{~km}, y=19.8-20.3 \mathrm{~km}$. For the $\alpha=4$, CEN case the maximum peak probability of 0.085 occurs in the box centered at $x=2.0 \mathrm{~km}, y=20.1 \mathrm{~km}$, while the secondary probability peak of value 0.072 occurs at $x=2.1 \mathrm{~km}, y=20.3 \mathrm{~km}$. The 90 percent probability contour is enclosed within the area 
$x=1.75-2.3 \mathrm{~km}, y=19.8-20.5 \mathrm{~km}$. The reason for the double peaked probability distribution for the CEN backtrack method initial guess points in both the $\alpha=2$ and $\alpha=4$ cases is probably due to a secondary minimum in the $\mathrm{X}^{2}$ function. The outlying CEN initial guess point distribution is apparently forcing the inversion program to choose this secondary minimum quite often.

The procedure for finding the most probable release time and source strength is to determine the maximum likelihood of these quantities for all inversions which occur within the $x-y$ square having the highest location probability. This is done by selecting bins in $t$ and $Q$ and plotting the histograms of the numbers of source location points with $t$ and $Q$ values in each bin of the $t$ and $Q$ range located in this $x-y$ square. Bin sizes were chosen at 10 seconds in time and $10 \mu \mathrm{g} / \mathrm{s}$ in $Q$. Examples of these histograms are shown in figures $5 \mathrm{a}(t)$ and $5 \mathrm{~b}(Q)$ for the $\alpha=2$, AV case. For the $\alpha=2, \mathrm{AV}$ and CEN cases, the most likely release time was between 3595 and 3605 seconds compared to the actual value of 3600 seconds, with maximum probabilities of $\sim 0.3$. For the $\alpha=4, \mathrm{AV}$ and CEN cases, the most likely release time was also between 3595 and 3605 seconds with maximum probabilities of $\sim 0.27$. For the $\alpha=2$, AV and CEN cases, the most likely source strength was between 95 and $105 \mu \mathrm{g} / \mathrm{s}$ compared to the actual value of $100 \mu \mathrm{g} / \mathrm{s}$ with maximum probabilities of 0.18 and 0.27 respectively. For the $\alpha=4, \mathrm{AV}$ and CEN cases, the most likely source strength was between 105 and $115 \mu \mathrm{g} / \mathrm{s}$ (compared to the actual value of $100 \mu \mathrm{g} / \mathrm{s}$ ) with maximum probabilities of $\sim 0.15$.

The results of the initial test calculations (Table 1) indicate that the inversion procedure for the initial test case produces good results with reasonably well defined maximum probabilities for the four source properties $(x, y, t$, and $Q)$ in a 1000 sensor realization scenario. The technique finds the source location, release time, and strength within $\sim \pm 50 \mathrm{~m}, \sim \pm 10 \mathrm{~s}$, and $\sim \pm 10 \mu \mathrm{g} / \mathrm{s}$ for the most probable values and within $\sim \pm 300 \mathrm{~m}, \sim \pm 25 \mathrm{~s}$, and $\sim \pm 45 \mu \mathrm{g} / \mathrm{s}$ at the $90 \%$ confidence level. The model runs relatively fast, taking $\sim 100$ seconds per inversion on a Sparc 10 workstation. The results presented above were obtained using the Sample 1 (random normal) method. A test calculation using the Sample 2 method (Monte Carlo sampling) will be discussed in a future report. Sensitivity calculations for the above examples relating to number of sensors, placement of sensors, times of sensor readings, wind field variations, and source strength will also be considered in a future report. It should be stressed that these calculations represent an initial step to evaluate an inversion package consisting of a Marquardt inversion model coupled to backtrack search and probability sampling algorithms and as such we chose to experiment with a simple puff release. More complex scenarios will have to deal with the effects of atmospheric turbulence on the statistics of the sensor readings, wind field uncertainty, nonGaussian plume dispersion (Ermak and Nasstrom, 2000), and rain out and regeneration effects of pollutants. The inclusion of these effects into a computationally efficient source inversion model may require a Bayesian approach to determine source properties. 
Table 1. Results Of Inversion Calculations For 1000 Sensor Realizations

\begin{tabular}{lcccc}
\hline & $x(\mathrm{~km})$ & $y(\mathrm{~km})$ & $t(\mathrm{sec})$ & $\mathrm{Q}(\mu \mathrm{g} / \mathrm{s})$ \\
\hline \hline True & 2.0 & 20.0 & 3600 & 100 \\
$\alpha=2$ AV & $1.95-2.05(0.34)^{*}$ & $19.95-20.05$ & $3595-3605(0.29)$ & $95-105(0.18)$ \\
$\alpha=2$ CEN & $1.95-2.05(0.23)$ & $19.95-20.05$ & $3585-3595(0.30)$ & $95-105(0.27)$ \\
$\alpha=4$ AV & $1.95-2.05(0.10)$ & $19.95-20.05$ & $3595-3605(0.27)$ & $105-115(0.14)$ \\
$\alpha=4$ CEN & $1.95-2.05(0.09)$ & $20.05-20.15$ & $3595-3605(0.26)$ & $105-115(0.16)$ \\
\hline
\end{tabular}

* The probability shown in the $x$ column applies to the $x-y$ square shown in the table. 


\section{APPENDIX A. Details of the Marquardt Scheme}

The Levenberg-Marquardt algorithm is a standard method for fitting equations to data when the dependence of the fitted equation on one or more coefficients is nonlinear (Bevington and Robinson, 1992). It combines, in a smoothly varying manner, the efficiency of expanding the error cost function $\chi^{2}$ as a quadratic functional in the vicinity of its minimum with the more robust behavior of a steepest descent away from such a minimum. However, a numerical problem can occur when the fitted equation becomes independent of some parameters when another parameter approaches zero. As an example, for

$$
\hat{y}_{0}(x)=a_{0}+a_{1} e^{-a_{2} x}
$$

the value of $y_{0}(x)$ becomes independent of $a_{2}$ as $a_{1}->0$. This leaves $a_{2}$ completely unconstrained by a cost functional depending only on the evaluation of the fit, and can result in numerical overflow problems during the fitting process. As one solution to this problem, a minimum norm constraint on the coefficients can be added to the cost functional.

Consider a variation of the fit about that given by the initial estimate of its coefficients, $a$,

$$
\hat{y}(x)=\hat{y}_{0}(x)+\frac{\partial y_{0}}{\partial a} \delta a
$$

where $\hat{y}_{0}, a$, and $\delta a$ are considered to be column vectors of lengths $\mathrm{N}, \mathrm{M}$ and $\mathrm{M}$, respectively. Using Equation (2), we can write a modified $\chi^{2}$ as

$$
\chi^{2}=\left(y-\hat{y}_{0}-\frac{\partial y_{0}}{\partial a} \delta a\right)^{T} W\left(y-\hat{y}_{0}-\frac{\partial y_{0}}{\partial a} \delta a\right)+(a+\delta a)^{T} \omega(a+\delta a)
$$

where, $W$ is an NxN weight matrix, such as the inverse of the error covariance matrix of $y$, and $\omega$ is a diagonal matrix of rank $M$ determining both a small amplitude and relative scaling for the minimum norm constraint on the coefficients. Differentiating Equation (3) and equating the result to zero, we obtain

$$
\begin{aligned}
\frac{1}{2} \frac{\partial \chi^{2}}{\partial \delta a} & =-\left(y-y_{0}\right)^{T} W \frac{\partial y_{0}}{\partial a}+{\frac{\partial y_{0}}{\partial a}}^{T} W \frac{\partial y_{0}}{\partial a} \delta a+\omega(a+\delta a) \\
& =-\left[\left(y-y_{0}\right)^{T} W \frac{\partial y_{0}}{\partial a}-\omega a\right]+\left[{\frac{\partial y_{0}}{\partial a}}^{T} W \frac{\partial y_{0}}{\partial a}+\omega\right] \delta a=0
\end{aligned}
$$

Adopting the notation of Bevington and Robinson (1992), we can write

with,

$$
\alpha \delta a=\beta
$$




$$
\alpha=\left[\frac{\partial y_{0}{ }^{T}}{\partial a} W \frac{\partial y_{0}}{\partial a}+\omega\right], \text { and } \beta=\left[\left(y-y_{0}\right)^{T} W \frac{\partial y_{0}}{\partial a}-\omega a\right]
$$

Only the addition of the $\omega$ terms differentiates these from the standard definitions of $\alpha$ and $\beta$. Then, noting that the steepest descent method can be written as

$$
\delta a=\text { constant } * \beta
$$

where, $\lambda$ is a scalar. Defining

$$
\alpha^{\prime}=\alpha+\lambda \operatorname{diag}(a)
$$

we arrive at the Levenberg-Marquardt solution

$$
\alpha^{\prime} \delta a=\beta
$$

but with the additional minimum norm constraint included. When $\lambda$ is large, $\alpha^{\prime}$ becomes diagonally dominant and the algorithm tends towards the steepest descent method. When $\lambda$ is small, the algorithm tends towards the gradient expansion method. For a nominally small value of $\omega$, the additional norm constraint prevents $\alpha^{\prime}$ from becoming singular in regions of the coefficient space in which Equation (2) becomes independent of one or more coefficients.

As a further practical issue, the magnitude of the individual coefficients could vary by orders of magnitude. This provides the motivation for further scaling Equation (9) into

$$
\begin{aligned}
\left(c \alpha^{\prime} c\right)\left(c^{-1} \delta a\right) & =c \beta, \quad \text { or } \\
\tilde{\alpha} \delta \tilde{a} & =\tilde{\beta}
\end{aligned}
$$

where $c$ is a diagonal MxM matrix whose elements $\mathrm{c}_{\mathrm{ii}}$ are given by

$$
c_{i i}=\left[a_{i i}+\varepsilon M^{-1} \operatorname{trace}(a)+\varepsilon^{2}\right]^{-1}
$$

Thus $c$ allows for numerically nonsingular scaling whether a single diagonal element of $a$ is zero or in the degenerate case when all elements are zero.

Bevington and Robinson (1992) attribute the following recipe to Marquardt (1963)

1. Compute $\chi^{2}(a)$.

2. Start initially with $\lambda=0.001$.

3. Compute $\delta a$ and $\chi^{2}(a+\delta a)$ with this choice of $\lambda$.

4. If $\chi^{2}(a+\delta a)>\chi^{2}(a)$, increase $\lambda$ by a factor of 10 and repeat step 3 .

5. If $\chi^{2}(a+\delta a)<\chi^{2}(a)$, decrease $\lambda$ by a factor of 10 , consider $a^{\prime}=a+\delta a$ to be the new starting point, and return to step 3 , substituting $a^{\prime}$ for $a$. 
This recipe essentially starts by trying to solve the problem by the gradient expansion method and backtracks to using the less efficient steepest descent algorithm only when the initial attempt fails. Unfortunately, in highly nonlinear problems such as the fitting of coefficients within exponents, the initial attempts via gradient expansion may result in failure by numerical overflow. We have therefore adopted the much more conservative approach of starting with $\lambda=128$, and increasing or decreasing $\lambda$ by factors of 2 . We also take $\varepsilon^{2}$ to be the numerical resolution (e.g. $\mathrm{O}\left(10^{-16}\right)$ for double precision) and can use $\omega=\varepsilon^{0.75}$ in implementing the minimum norm constraint. 


\section{REFERENCES}

Barker, A. L., D. E. Brown, and W. N. Martin, 1995: Bayesian estimation and the Kalman filter., Computers Math. Applic., 30, pp. 55-77.

Bevington, P. R., and D. K. Robinson, 1992: Data reduction and error analysis for the physical sciences, $2^{\text {nd }}$ ed. Mcgraw-Hill Inc., New York, "BR".

Edwards, L. L., R. P. Freis, L. G. Peters, and P. H. Gudiksen, 1992: The use of nonlinear regression analysis for integrating pollutant measurements with atmospheric dispersion modeling for source term estimation. Nucl. Technol., 101, 168.

Ermak, D.L., and J.S. Nasstrom, 2000: A Lagrangian Stochastic Diffusion Method for Inhomogeneous Turbulence, Atmos. Environ., 34, 7, 1059-1068.

Flesch, T. K., J. D. Wilson, and E. Yee, 1995: Backward-Time Lagrangian stochastic dispersion models and their application to estimate gaseous emissions., J. Appl. Meteor., 34, pp 13201332.

Hodur, R, M., 1997: The Naval Research Laboratory's coupled ocean/atmosphere mesoscale prediction system (COAMPS). Mon. Wea. Rev., 119, 1414-1430.

Iman, R. L., and M. J. Shortencarier, 1984: A FORTRAN 77 program and user's guide for the generation of Latin Hypercube and random samples for use with computer models. SNL Report NUREG/CR-3624, SAND83-2365.

Kasibhatla, P., M. Heimann, P. Rayner, N. Mahowald, R. G. Prinn, and D. Hartley, eds., 2000: Inverse methods in global biogeochemical cycles. American Geophysical Union, Washington D. C.

Leach, M. J. and H. N. Chin, 2000: Uncertainty in dispersion forecasts using meteorological ensembles. In prep. For Atmos. Env.

Marquardt, D.W., 1963: An Algorithm for Least-Squares Estimation of Nonlinear Parameters, J. Soc. Ind. Appl. Math., Vol. II, No. 2, pp. 431-441.

Petersen, W. B., and L. Lavadas, 1986: INPUFF 2.0 - A multible source Gaussian puff dispersion algorithm. EPA Report EPA-600/8-86/024.

Yee, E., P. R. Kosteniuk, and J. F. Bowers, 1998: A study of concentration fluctuations in instantaneous clouds in the atmospheric surface layer for relative turbulent diffusion: basic descriptive statistics. Boundary-Layer Meteorology, 87, pp 409-457. 


\section{FIGURE CAPTIONS}

Figure 1. The COAMPS model generated wind vector field over the $30 \mathrm{~km} \times 20 \mathrm{~km}$ domain superimposed on a map of the one hour integrated pollutant concentrations for the actual source ( $x=2 \mathrm{~km}, y=20 \mathrm{~km}, t=3600 \mathrm{~s}, Q=100 \mu \mathrm{g} / \mathrm{s}$ ) calculated by the INPUFF model for a hi-resolution sensor field spacing of $x=1 \mathrm{~km}$ and $y=1 \mathrm{~km}$.

Figure 2a. The estimated source location points $(x, y)$ for 1000 concentration realizations with $\alpha$ $=2$, Sample 1 methodology. The " + " signs are the locations obtained using the concentration weighted average backtrack method (CEN) and the " $x$ " signs are the locations using the straight average backtrack method (AV).

Figure $2 b$. The estimated source location points $(x, y)$ for 1000 concentration realizations with $\alpha$ $=4$, Sample 1 methodology. The " + " signs are the locations obtained using the concentration weighted average backtrack method (CEN) and the " $x$ " signs are the locations using the straight average backtrack method (AV).

Figure 3a. The AV source location probability histogram (number of source locations) in each $0.1 \mathrm{~km}$ square of $x-y$ space for the $\alpha=2$ case.

Figure $3 b$. The CEN source location probability histogram (number of source locations) in each $0.1 \mathrm{~km}$ square of $x-y$ space for the $\alpha=2$ case.

Figure 3c. The AV source location probability histogram (number of source locations) in each $0.1 \mathrm{~km}$ square of $x-y$ space for the $\alpha=4$ case.

Figure 3d. The CEN source location probability histogram (number of source locations) in each $0.1 \mathrm{~km}$ square of $x-y$ space for the $\alpha=4$ case.

Figure $4 \mathrm{a}$. The AV source location probability histogram contour plot (number of source locations) in each $0.1 \mathrm{~km}$ square of $x-y$ space for the $\alpha=2$ case.

Figure $4 \mathrm{~b}$. The CEN source location probability histogram contour plot (number of source locations) in each $0.1 \mathrm{~km}$ square of $x-y$ space for the $\alpha=2$ case.

Figure 4c. The AV source location probability histogram contour plot (number of source locations) in each $0.1 \mathrm{~km}$ square of $x-y$ space for the $\alpha=4$ case.

Figure 4d. The CEN source location probability histogram contour plot (number of source locations) in each $0.1 \mathrm{~km}$ square of $x-y$ space for the $\alpha=4$ case.

Figure 5a. The release time probability histogram (numbers of source location points in each bin of the $t$ range located in the $x-y$ square having maximum location probability) for the $\alpha=2$ AV case. Bin size was chosen at 10 seconds in time.

Figure $5 \mathrm{~b}$. The source strength probability histogram (numbers of source location points in each bin of the $\mathrm{Q}$ range located in the $x-y$ square having maximum location probability) for the $\alpha=2 \mathrm{AV}$ case. Bin size was chosen at $10 \mu \mathrm{g} / \mathrm{s}$ in $\mathrm{Q}$. 


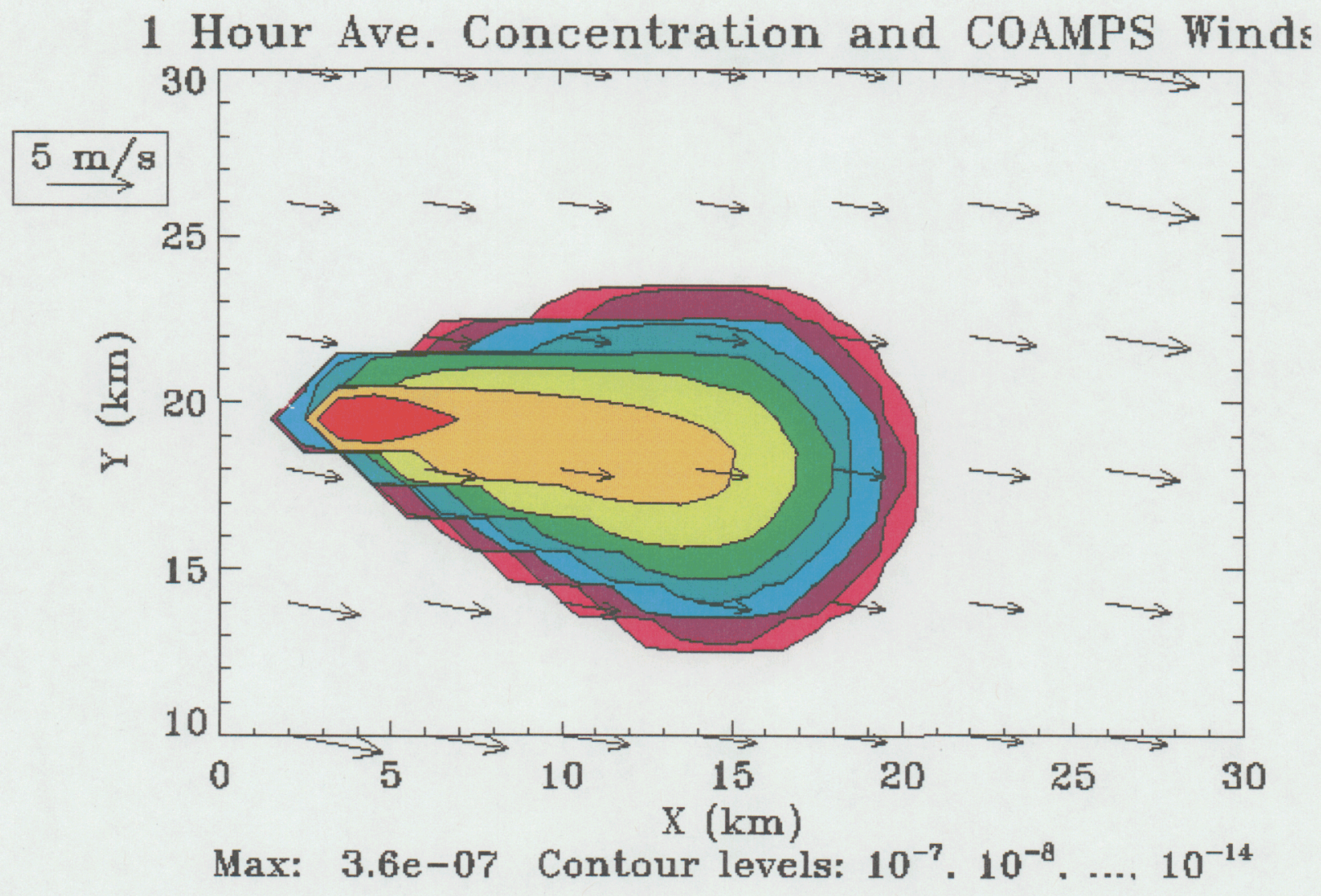

Figure 1. The COAMPS model generated wind vector field over the $30 \mathrm{~km} \times 20 \mathrm{~km}$ domain superimposed on a map of the one hour integrated pollutant concentrations for the actual source $(x=2 \mathrm{~km}, y=20 \mathrm{~km}, t=3600 \mathrm{~s}, Q=100 \mu \mathrm{g} / \mathrm{s})$ calculated by the INPUFF model for a hi-resolution sensor field spacing of $x=1 \mathrm{~km}$ and $y=1 \mathrm{~km}$. 


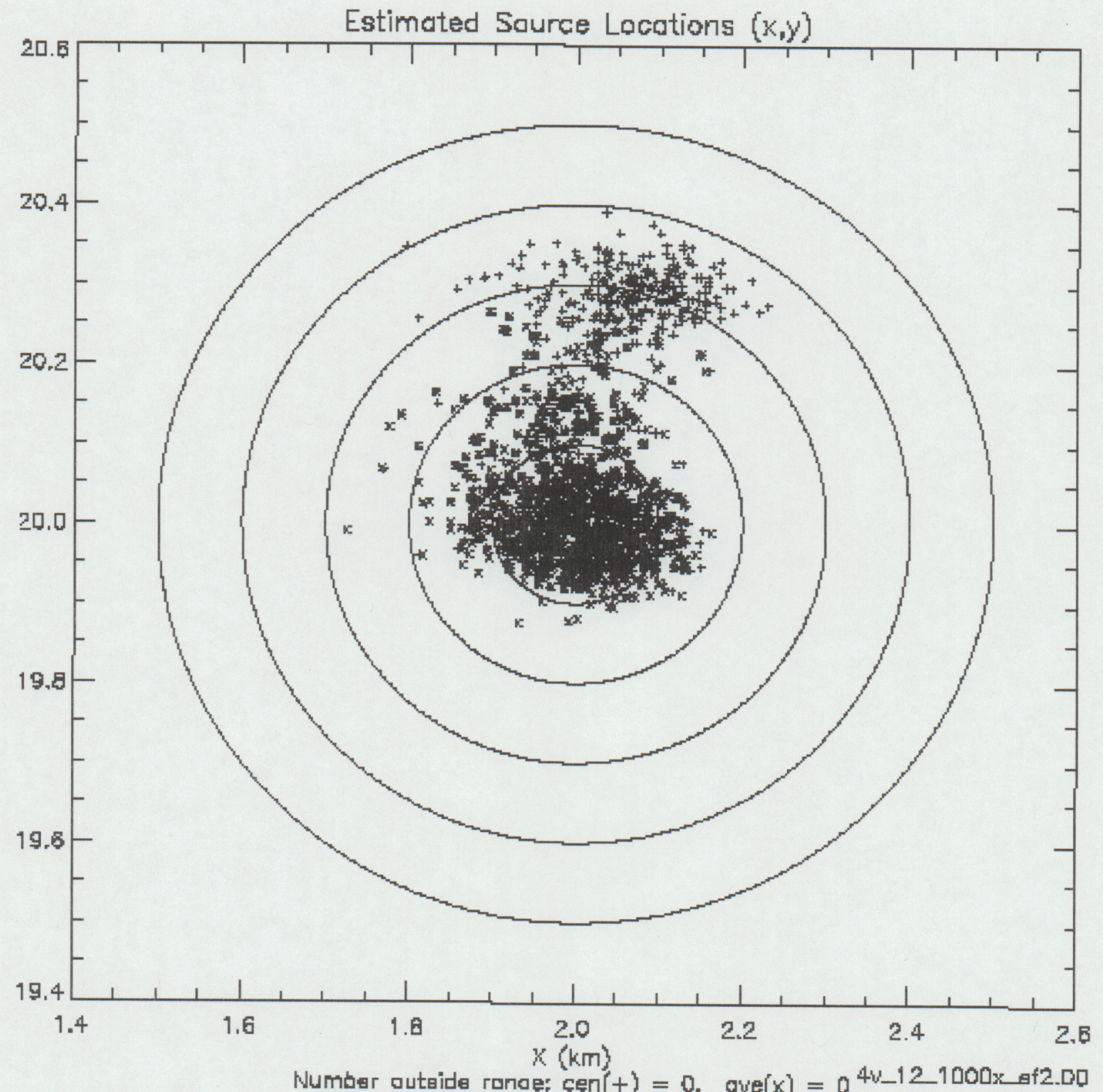

Figure 2a. The estimated source location points $(x, y)$ for 1000 concentration realizations with $\alpha$ $=2$, Sample 1 methodology. The " + " signs are the locations obtained using the concentration weighted average backtrack method (CEN) and the " $x$ " signs are the locations using the straight average backtrack method (AV). 
Estimated Source Locations $(x, y)$

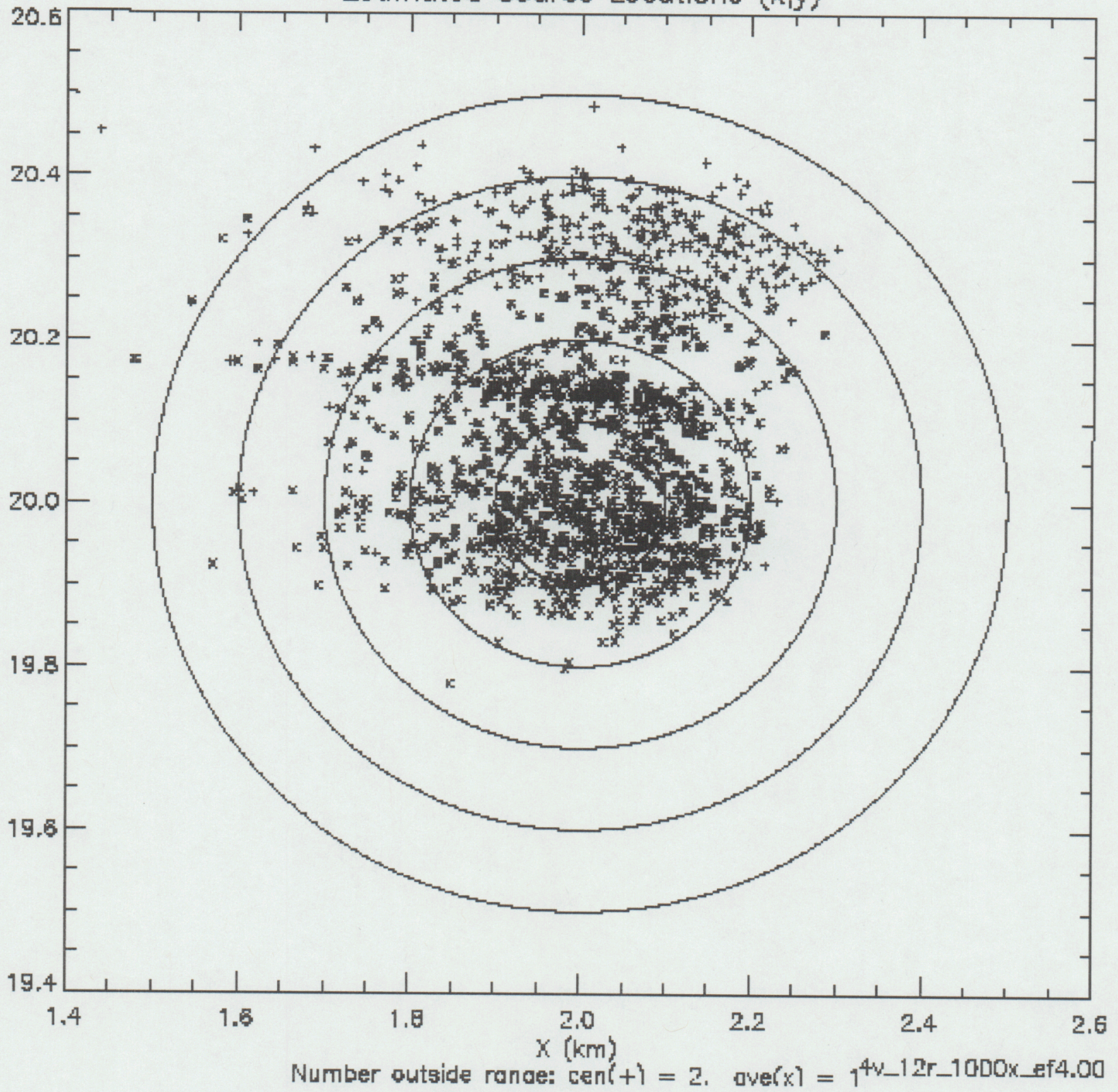

Figure $2 \mathrm{~b}$. The estimated source location points $(x, y)$ for 1000 concentration realizations with $\alpha$ $=4$, Sample 1 methodology. The "+" signs are the locations obtained using the concentration weighted average backtrack method (CEN) and the " $x$ " signs are the locations using the straight average backtrack method (AV). 


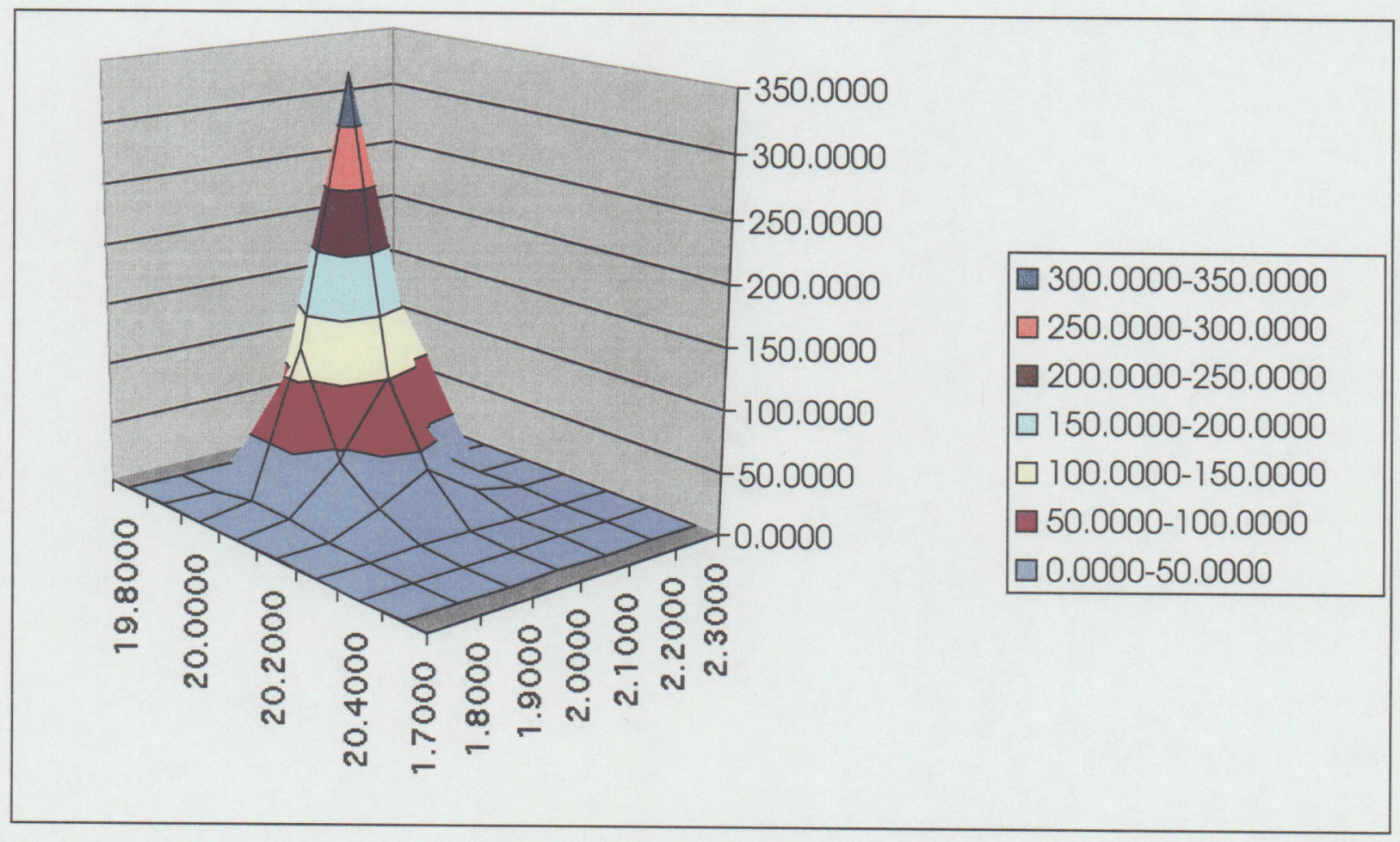

Figure 3a. The AV source location probability histogram (number of source locations) in each $0.1 \mathrm{~km}$ square of $x-y$ space for the $\alpha=2$ case. 


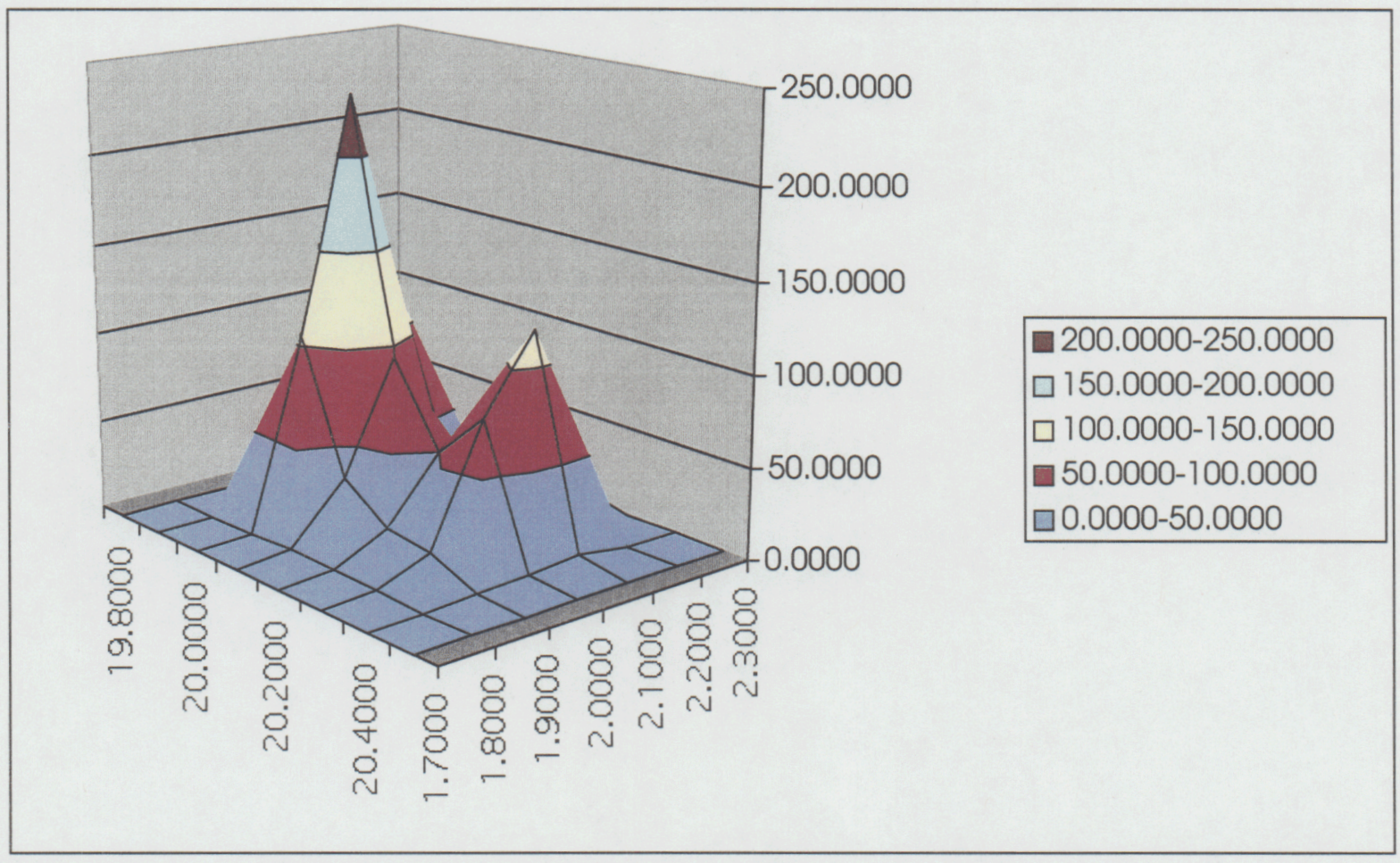

Figure $3 \mathrm{~b}$. The CEN source location probability histogram (number of source locations) in each $0.1 \mathrm{~km}$ square of $x-y$ space for the $\alpha=2$ case. 


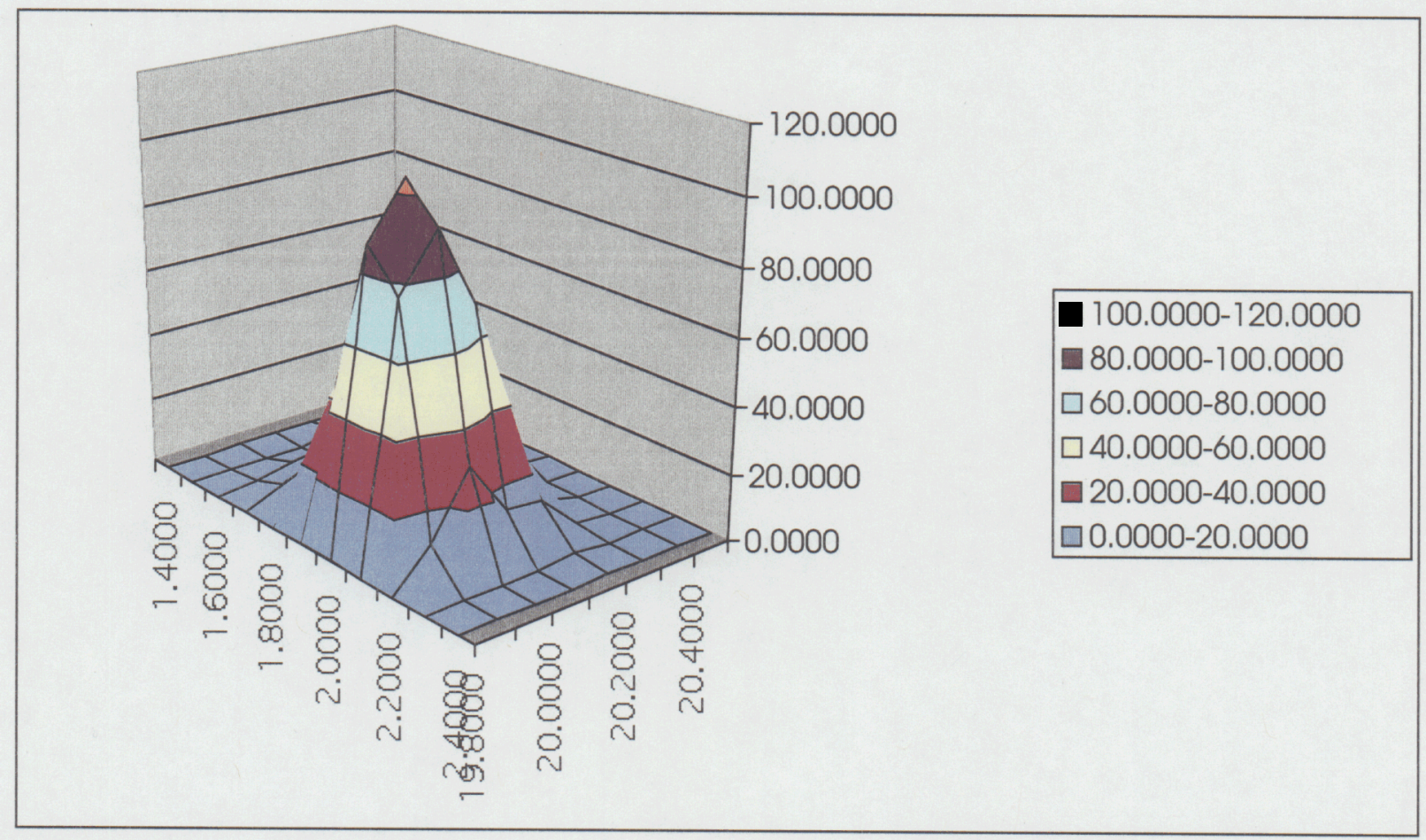

Figure 3c. The AV source location probability histogram (number of source locations) in each $0.1 \mathrm{~km}$ square of $x-y$ space for the $\alpha=4$ case. 


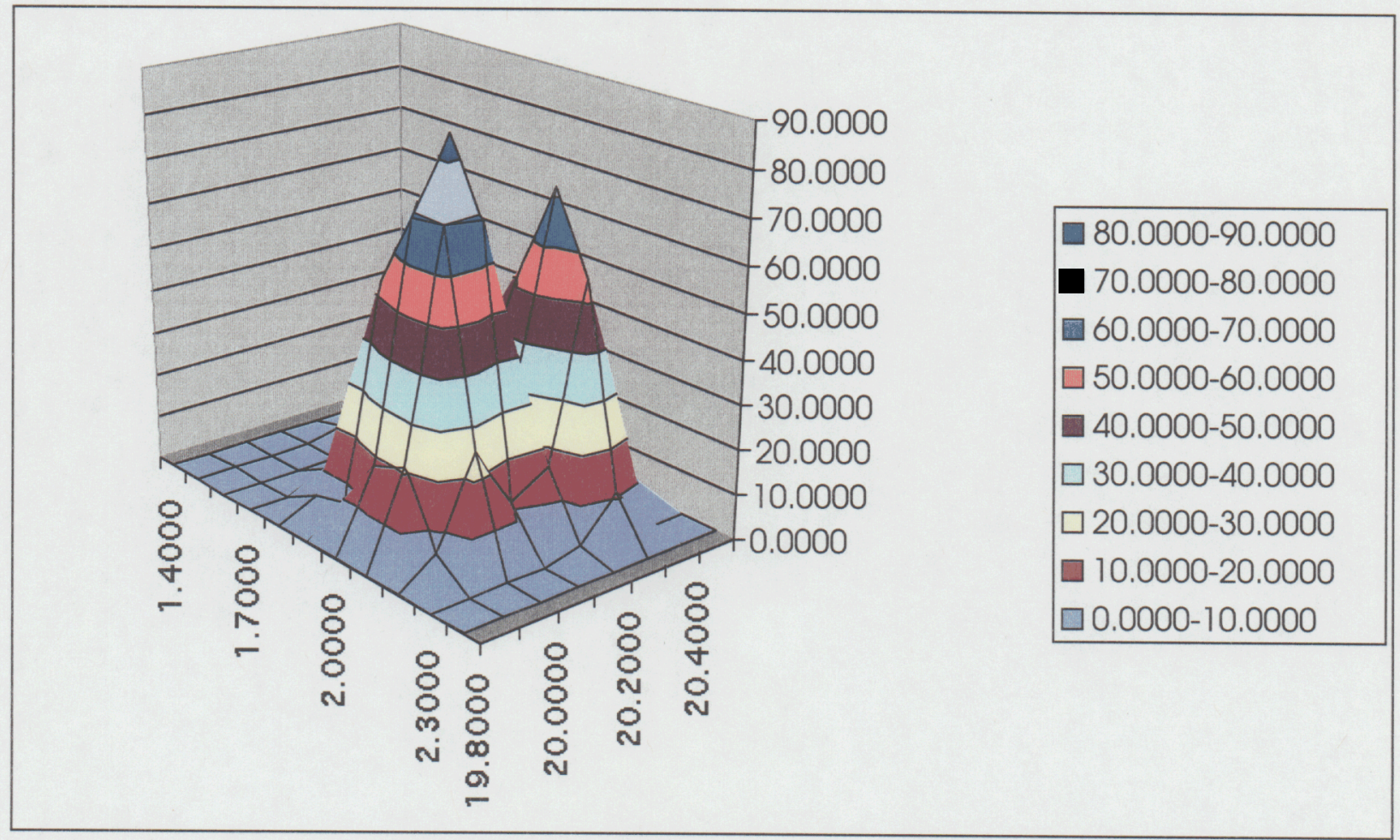

Figure 3d. The CEN source location probability histogram (number of source locations) in each $0.1 \mathrm{~km}$ square of $x-y$ space for the $\alpha=4$ case. 


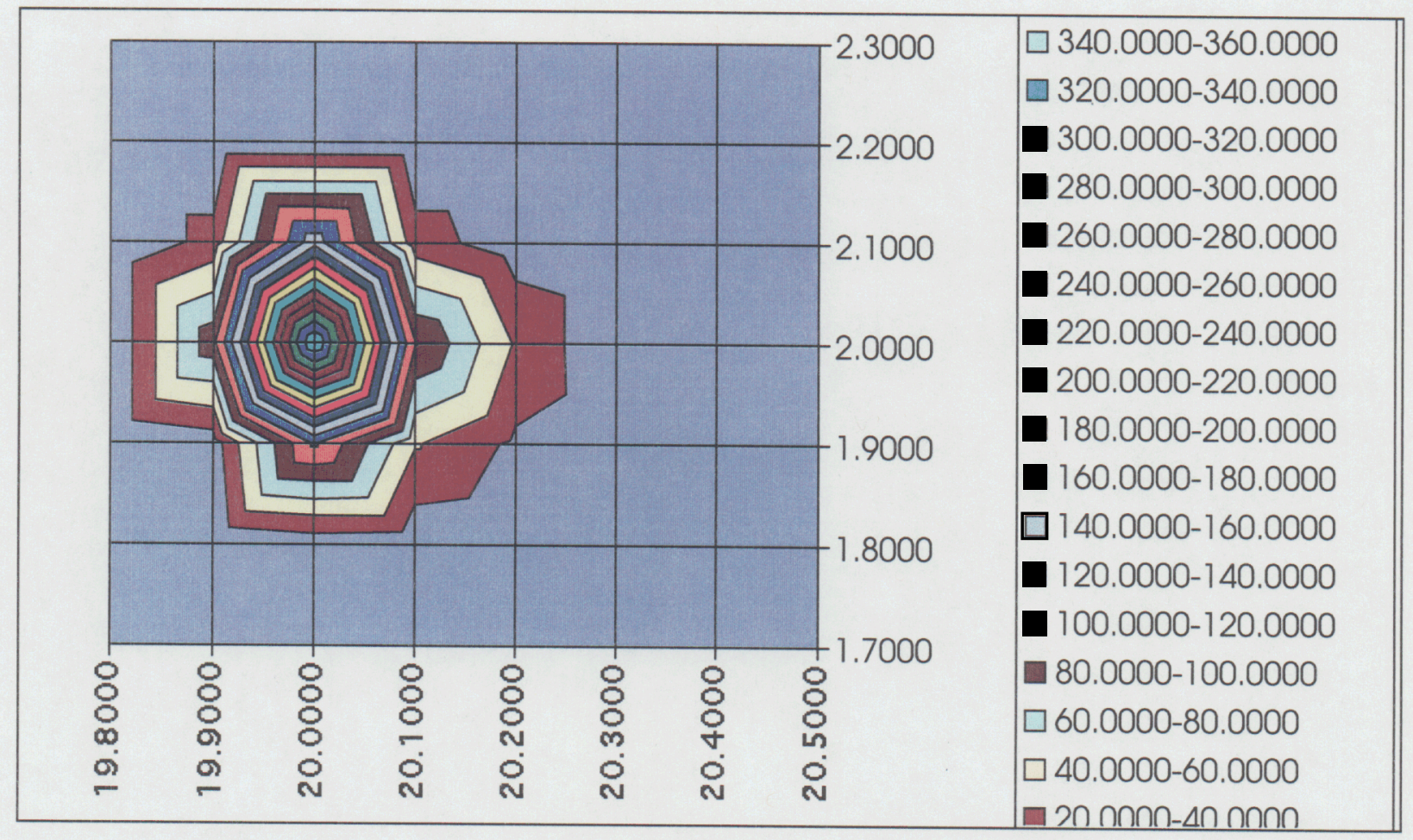

Figure 4a. The AV source location probability histogram contour plot (number of source locations) in each $0.1 \mathrm{~km}$ square of $x-y$ space for the $\alpha=2$ case. 


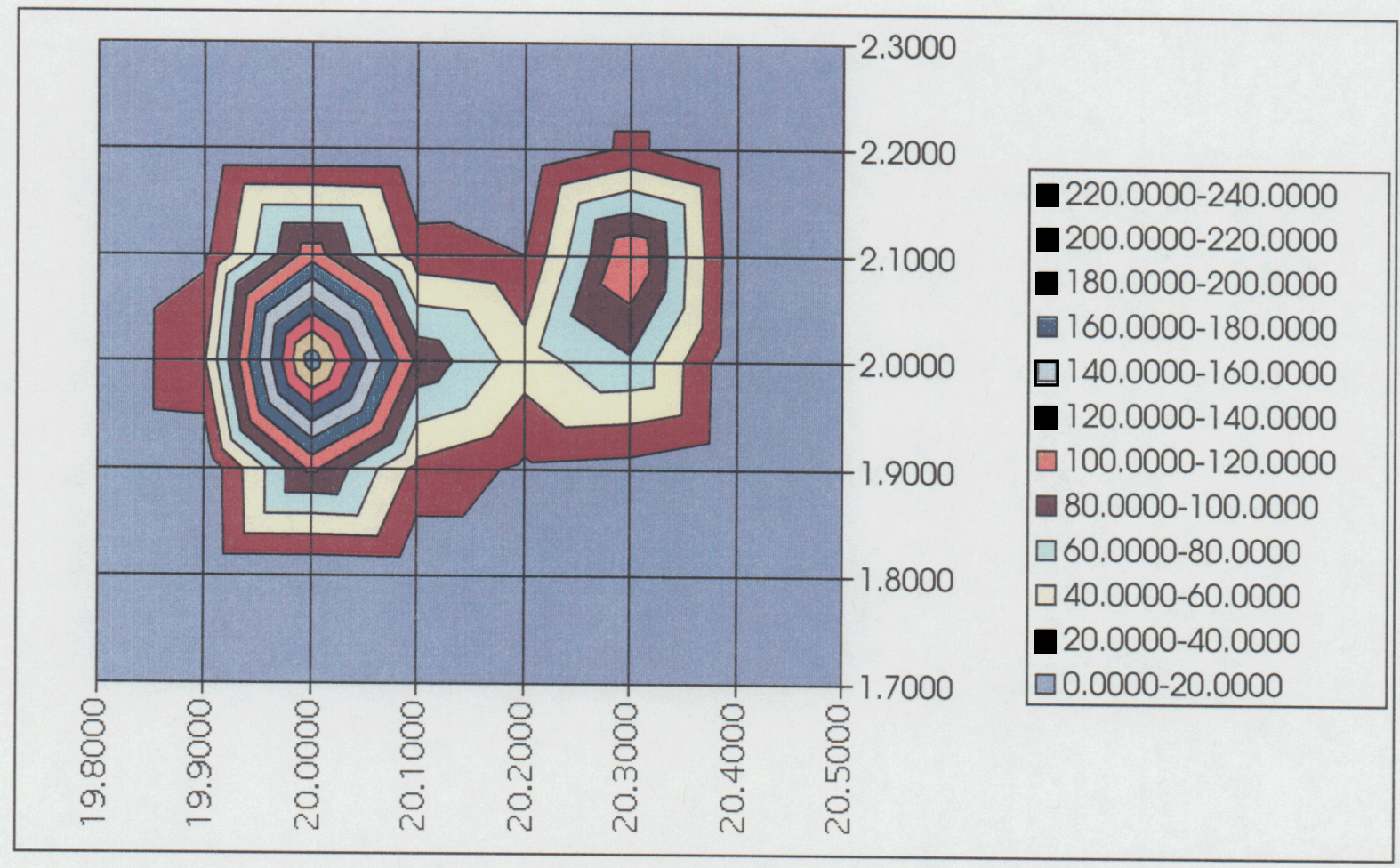

Figure 4b. The CEN source location probability histogram contour plot (number of source locations) in each $0.1 \mathrm{~km}$ square of $x-y$ space for the $\alpha=2$ case. 


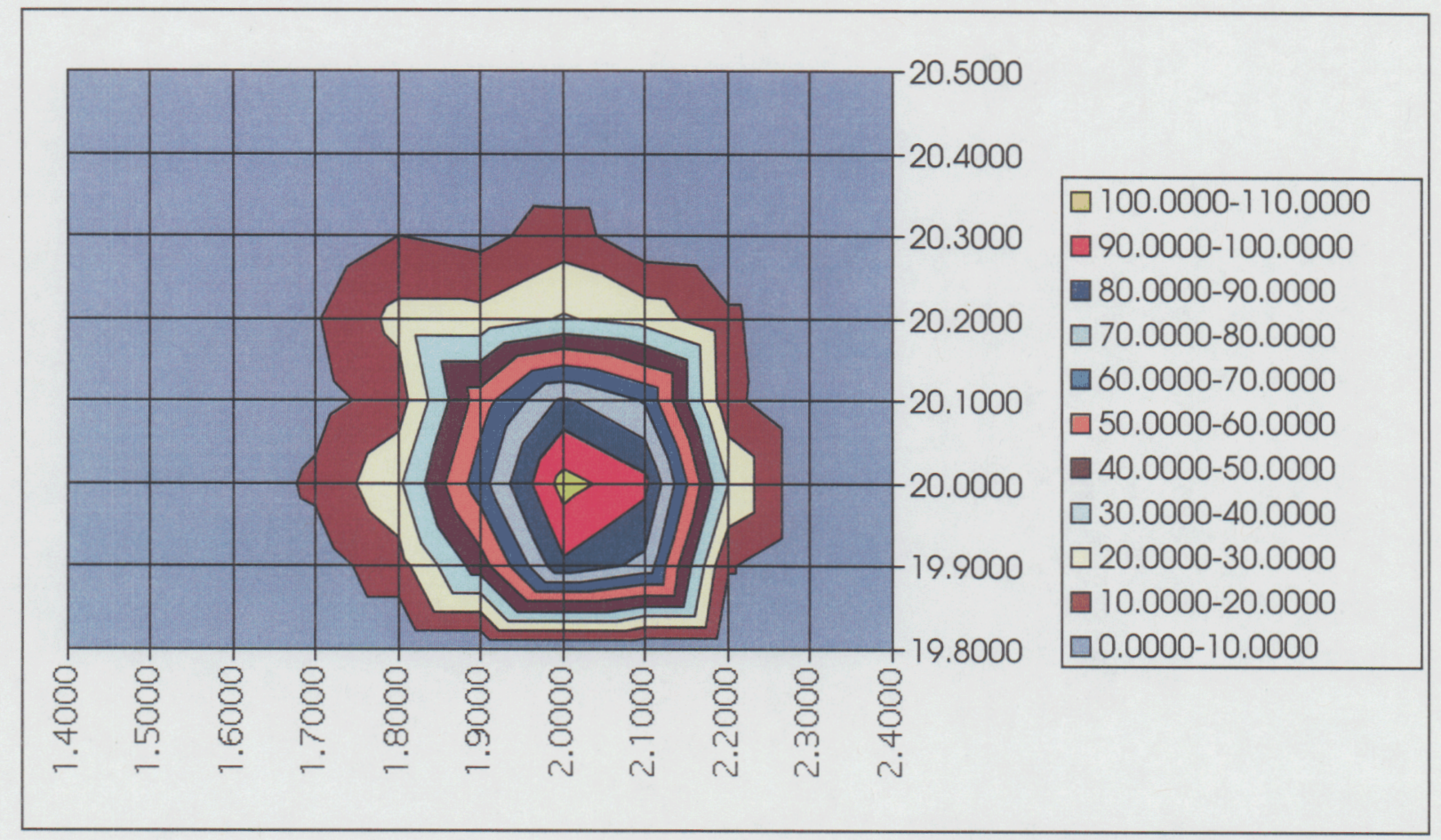

Figure 4c. The AV source location probability histogram contour plot (number of source locations) in each $0.1 \mathrm{~km}$ square of $x-y$ space for the $\alpha=4$ case. 


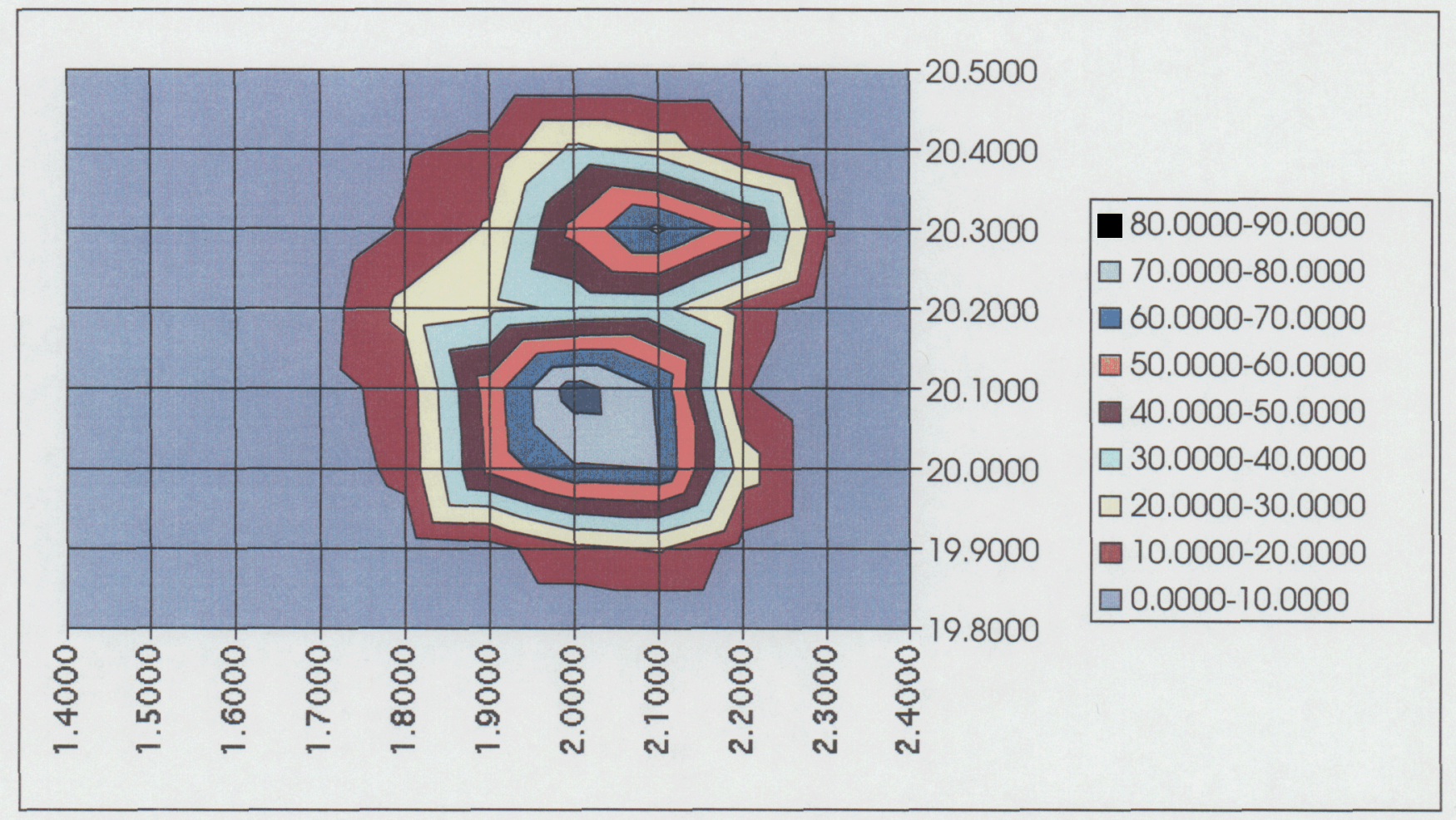

Figure 4d. The CEN source location probability histogram contour plot (number of source locations) in each $0.1 \mathrm{~km}$ square of $x-y$ space for the $\alpha=4$ case. 


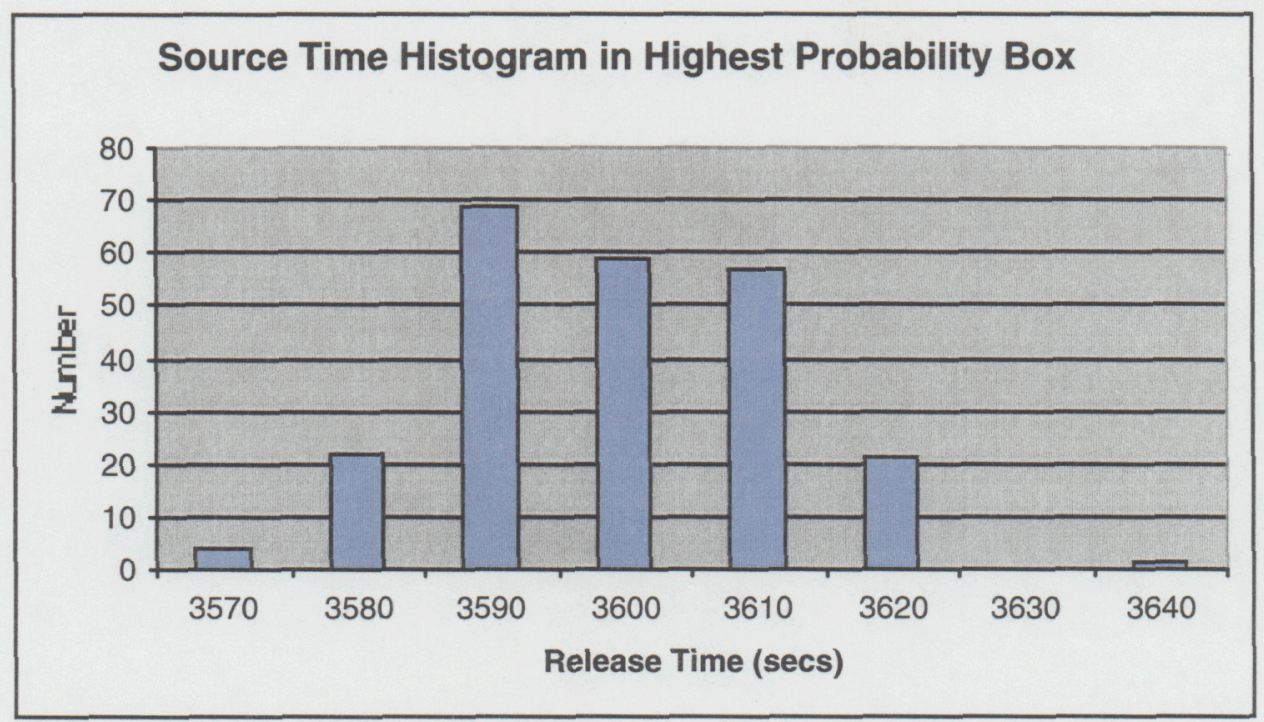

Figure 5a. The release time probability histogram (numbers of source location points in each bin of the $t$ range located in the $x$ - $y$ square having maximum location probability) for the $\alpha=2$ AV case. Bin size was chosen at 10 seconds in time.

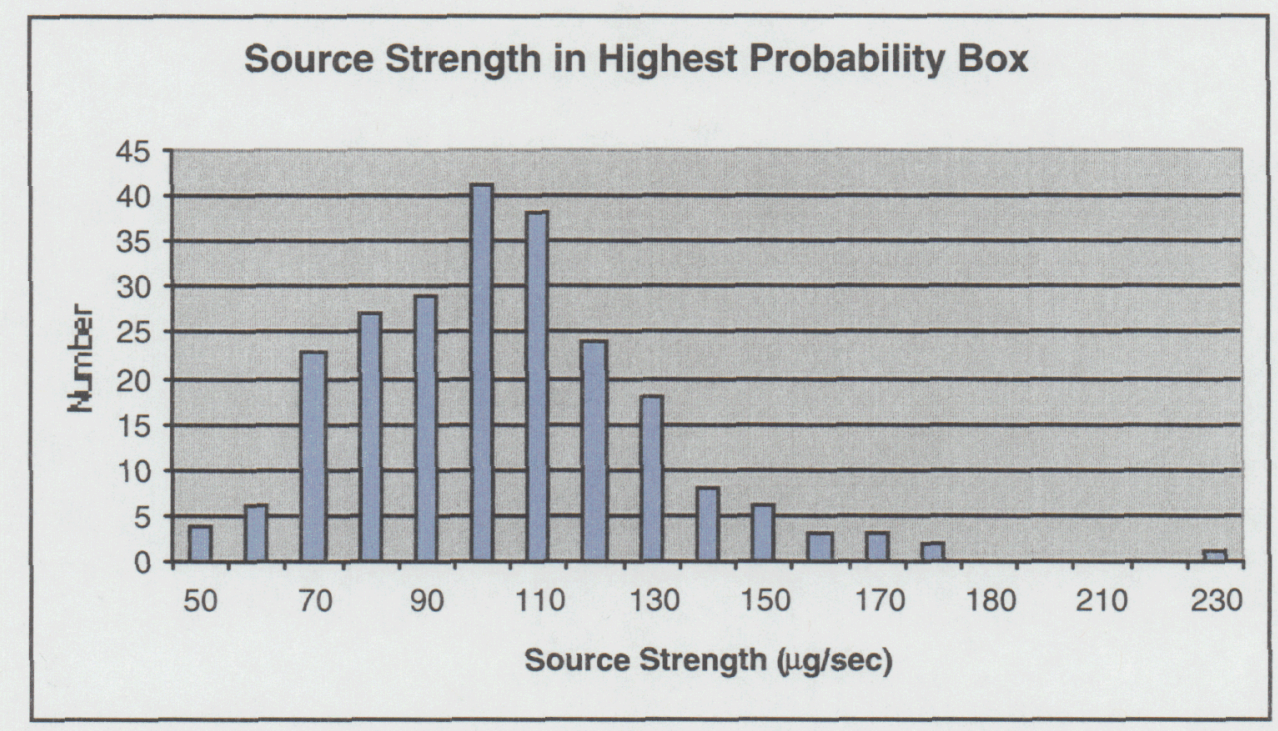

Figure 5b. The source strength probability histogram (numbers of source location points in each bin of the $Q$ range located in the $x-y$ square having maximum location probability) for the $\alpha=2 \mathrm{AV}$ case. Bin size was chosen at $10 \mu \mathrm{g} / \mathrm{s}$ in $\mathrm{Q}$. 